\title{
Space from string bits
}

\section{Charles B. Thorn}

Institute for Fundamental Theory, Department of Physics, University of Florida, 2001 Museum Road, Gainesville, FL 32611-8440, U.S.A.

E-mail: thorn@phys.ufl.edu

ABSTRACT: We develop superstring bit models, in which the lightcone transverse coordinates in $D$ spacetime dimensions are replaced with $d=D-2$ double-valued "flavor" indices $x^{k} \rightarrow f_{k}=1,2 ; k=2, \ldots, d+1$. In such models the string bits have no space to move. Letting each string bit be an adjoint of a "color" group $\mathrm{U}(N)$, we then analyze the physics of 't Hooft's limit $N \rightarrow \infty$, in which closed chains of many string bits behave like free lightcone IIB superstrings with $d$ compact coordinate bosonic worldsheet fields $x^{k}$, and $s$ pairs of Grassmann fermionic fields $\theta_{L, R}^{a}, a=1, \ldots, s$. The coordinates $x^{k}$ emerge because, on the long chains, flavor fluctuations enjoy the dynamics of $d$ anisotropic Heisenberg spin chains. It is well-known that the low energy excitations of a many-spin Heisenberg chain are identical to those of a string worldsheet coordinate compactified on a circle of radius $R_{k}$, which is related to the anisotropy parameter $-1 \leq \Delta_{k} \leq 1$ of the corresponding Heisenberg system. Furthermore there is a limit of this parameter, $\Delta_{k} \rightarrow \pm 1$, in which $R_{k} \rightarrow \infty$. As noted in earlier work [Phys. Rev. D 89 (2014) 105002], these multi-string-bit chains are strictly stable at $N=\infty$ when $d<s$ and only marginally stable when $d=s$. (Poincaré supersymmetry requires $d=s=8$, which is on the boundary between stability and instability.)

Keywords: M(atrix) Theories, Bethe Ansatz

ARXIV EPRINT: 1407.8144 


\section{Contents}

1 Introduction $\quad 1$

2 Superstring bit models $\quad 4$

3 Emergence of Grassmann and longitudinal space $\quad 8$

4 Heisenberg spin chain $\quad 9$

$\begin{array}{lr}4.1 \text { Energy analysis for } \Delta=0 & 10\end{array}$

$\begin{array}{lll}4.2 & \text { Lowest lying energies of } H^{\prime}=-H_{\mathrm{Hei}} & 11\end{array}$

$5 \quad$ Emergence of transverse space $\quad 13$

6 Conclusion $\quad 14$

A Energy analysis for $-1<\Delta<1$, and large $M \quad 16$

$\begin{array}{lll}\text { A.1 Consecutive } I_{l}: Q, P \neq 0 & 16\end{array}$

A.2 Non-consecutive $I_{l}$

\section{Introduction}

The idea, that string bits might provide the fundamental constituents of string, was proposed over two decades ago [1], with the implications of supersymmetry for string bit models developed and explored in [2, 3]. As initially envisioned, string bits were point particles moving about in the transverse space $x^{k}, k=2, \ldots, D-1$ of lightcone coordinates, $x^{ \pm}=\left(x^{0} \pm x^{1}\right) / \sqrt{2}$ enjoying a dynamics that is Galilei invariant. This Galilei invariance is natural to lightcone coordinates in which the momentum component $P^{+}=\left(p^{0}+p^{1}\right) / \sqrt{2}$ plays the role of a variable Newtonian mass, and the Galilei transformations act on the transverse space $x^{k} \rightarrow x^{k}+V^{k} x^{+}$. The Newtonian mass $m$ of each string bit is fixed, but then Galilei invariance ensures that the Newtonian mass of a bound state of $M$ string bits is $M m$. If bound states can form with any number $M$ of bits, $M m$ can be interpreted as the total (discretized) $P^{+}$of the bound state. For $M \rightarrow \infty$ this emergent $P^{+}$can be regarded as a continuous variable whose conjugate can be interpreted as $x^{-}$. String theory emerges from these models, if the many-bit bound states are closed linear chains of string bits (which can be arranged in the context of the 't Hooft large $N$ limit $[4,5]$ ), in which the low lying excitation energies scale for $M \rightarrow \infty$ as $M^{-1}$. This scaling law then leads to a Poincaré invariant dispersion relation $P^{-}=\left(\boldsymbol{P}^{2}+\mu^{2}\right) /\left(2 P^{+}\right)$. Since space is 3 dimensional with the associated transverse space 2 dimensional, the string bit model provided a concrete realization of 't Hooft's idea that the world is like a hologram [6-8]. 
Recently Sun and I have begun a new study of string bit models in a more general context [9]. Our main idea is that relaxing the strict requirement, that super Poincaré invariance emerge, provides us with composite models of string without the infrared instabilities caused by the massless graviton and gauge particles of superstring theory. Specifically, the lightcone quantized type II superstring requires a worldsheet system of 8 bosonic coordinate fields $x^{k}$ and 8 pairs of fermionic Grassmann fields $\theta_{L, R}^{a}$. We proposed studying general string bit models in which $d$ bosonic and $s$ pairs of fermionic worldsheet fields emerge, provided that $d<s$. As long as $d<s$ the emergent closed string ground state has positive mass squared $\left(2 P^{+} P^{-}-\boldsymbol{p}^{2}>0\right)$, implying that the lowest energy closed string chain has its number of bits $M \rightarrow \infty$, i.e. it behaves as a continuous string. The superstring, with $d=s=8$, is only marginally stable, and when $d>s$ long closed chains are unstable and will not form. As a case in point we began studying the simplest stable case in which the emergent string has $d=0$ and $s=1$ with further analysis of this model to be given in [10].

In this paper I would like to extend the work of [9] in a different direction by developing some of the string bit models, proposed in that work, which lead to composite string with general $d<s$. The models with $d=0$ and general $s>0$ can be obtained from $[2,3]$ by simply discarding the dependence on transverse coordinates. Then each string bit is an adjoint in $\mathrm{U}(N)$ color and has $2^{s}$ spin states. Half of these are bosons and half fermions. Of course, for $s=1$ the model reduces to that studied in [9], with one boson and one fermion. To achieve $d>0$ we could simply follow $[2,3]$ and restore the dependence on transverse coordinates. Instead, as already suggested in [9], we let each string bit have $2^{d}$ "flavor states". Then all together, each string bit will have $2^{s} 2^{d} N^{2}$ internal states. It is only when these bits form long closed chains that fluctuations among the internal bit states begin to behave like bosonic and fermionic coordinates. Then we can say that space has effectively emerged from string bit dynamics - hence the title chosen for this paper.

Although string theory and the dual resonance models, which led to its discovery, were initially developed as models of extended objects moving in space, it has been understood, almost from the beginning, that the "target space" in which string "moves" need not be a continuous manifold. Indeed, once one posits the existence of a worldsheet, the target space can be any two dimensional quantum field theory, which supports a suitable Virasoro algebra with the appropriate central charge (conformal field theories). The original description of the evolving string as a mapping $x^{\mu}(\sigma, \tau)$ from a worldsheet parameterized by $\sigma, \tau$ to space time $x^{\mu}$ could in the extreme be replaced by a mapping to $x^{ \pm}(\sigma, \tau)$ and the values of a bunch of fermion fields $\psi_{a}(\sigma, \tau)$ on the worldsheet. ${ }^{1}$ By bosonizing some of these fermion worldsheet fields, one can regain (compact) coordinates. Thus string theory automatically provides a first step in understanding the concept of space as an emergent phenomenon.

The goal of string bit models is to understand the worldsheet itself as an emergent phenomenon. In this paper, we shall focus on models which generate a worldsheet in lightcone parameterization in which $x^{+}=\tau$ and $\sigma$ is chosen so the density of $P^{+}$is unity, $0<\sigma<P^{+}[12-15]$. To motivate them let's "deconstruct" the lightcone worldsheet path

\footnotetext{
${ }^{1} \mathrm{By}$ retaining $x^{+}$as a continuous one dimensional manifold one keeps conventional quantum dynamics, which requires the notion of a Hamiltonian $P^{-}$. But this does not seem to be absolutely mandatory, provided that one is prepared to replace quantum mechanics with something else [11].
} 
integral for a free closed string. First, the path integral is defined on a 2 dimensional lattice [16], which discretizes $\tau=x^{+} \rightarrow k b$ and $\sigma \rightarrow l m$. Then we pass to Hamiltonian quantum mechanics by sending $b \rightarrow 0$. This leaves us with a system of point particles, each carrying a single unit of $P^{+}=m$, ordered on a closed chain enjoying nearest neighbor interactions. Finally we embrace these string bits as fundamental degrees of freedom which are not a priori confined to closed chains. Instead of describing the string bits by their trajectories $\boldsymbol{x}_{l}(\tau)$, we introduce a string bit annihilation operator $a(\boldsymbol{x})$ and an empty state $|0\rangle$ [1]. A superstring bit [2,3] can be either a fermion or boson. And as already mentioned, the transverse space label $\boldsymbol{x}$ can be replaced by discrete internal symmetry labels. After this, as far as the string bits are concerned, space is literally nonexistent.

The emergence of the concept of space in these models depends on a remarkable confluence of circumstances involving string bit dynamics. They should be such that string bits organize themselves into closed many bit chains. The lowest energy chains must have either an infinite or at least an extremely large number of bits $M \gg 1$. Once chains of string bits form, the low-lying energy excitations of a chain will be "spin waves" involving fluctuations of the internal string bit states (including fluctuations of statistics!). For large $M$ these low excitation energies will naturally scale as $M^{-1}$. This leads to the interpretation of the chain energy eigenstate as a particle moving in one space dimension, with lightcone dispersion relation $P^{-}=\mu^{2} / 2 P^{+}$. The spectrum of particle masses $\mu^{2}$ depends on the nature of the internal fluctuation waves allowed by the dynamics. Thus the Hamiltonian giving string bit dynamics is interpreted as $P^{-}$and the bit number as $P^{+}=M m$. The longitudinal dimension $x^{-}$therefore emerges as the conjugate to $P^{+}$.

A straightforward way to set up the string bit dynamics to favor chain formation is to exploit the 't Hooft large $N$ expansion [4]. This is done [1,5] by letting the annihilation operator for a string bit be an $N \times N$ matrix $\left(a_{K}\right)_{\alpha}^{\beta}$, and choosing the Hamiltonian as a sum of terms with the structure

$$
\frac{2}{N} \operatorname{Tr} a_{K}^{\dagger} a_{L}^{\dagger} a_{I} a_{J}
$$

Then when $N \rightarrow \infty$ the Hamiltonian connects single trace states to single trace states with only nearest neighbor interactions, which sets up a one dimensional spin chain problem. ${ }^{2}$ In [9] we observed that long chains will be energetically favored if the number of statistics fluctuating waves $s$ exceeds the number of statistics nonfluctuating waves $d$. We then studied in great detail a model where there was precisely one of the former and none of the latter. For supersymmetry these two types of waves are equal in number, so the string bit model underlying superstring is on the boundary between stability and instability.

The string bit Hamiltonians we consider in this paper all have the general structure of (1.1): they are sums of terms quartic in the fields with two creation operators and two

\footnotetext{
${ }^{2}$ To be clear, all of the microscopic string bit models considered in this paper will have a large $N$ limit which describes free fundamental string with constant non-zero positive string tension $T_{0}$. This is achieved by tuning the parameters of the string bit model so that the low energy excitations of the large $N$ chain precisely match the excitations of the free string. These models should not be confused with models based on the AdS/CFT correspondence, in which the string on the AdS side has a dynamical tension which depends on the string's location in AdS space and vanishes far from the AdS boundary.
} 
annihilation operators. It is only for simplicity that we don't include many body terms like $\operatorname{Tr} a^{\dagger n} a^{n}$ with $n=3,4 \ldots$, which are allowed in principle. Two-body terms seem to be rich enough to provide for the emergence of space from string bits, at least in 't Hooft limit $N \rightarrow \infty$ analyzed in this paper.

In the next section 2 , we present the string bit models, which are studied in this paper. Then in section 3 we explain how long closed chains form dynamically and thereby convert fluctuating internal spin states to Grassmann worldsheet fields. In section 4 we discuss the Heisenberg spin chain. We present the Bethe ansatz [17] for its energy eigenstates for general anisotropy parameter $\Delta$. For $\Delta=0$ it is easy to read off the energy spectrum for $M$ spins and evaluate its large $M$ behavior. For $\Delta \neq 0$ Yang and Yang $[18,19]$ have analyzed the energy spectrum for general $\Delta$ and $M \gg 1$. We review their analysis in an appendix, with particular attention to the aspects relevant to the present paper. Then in section 5 we explain how the formation of long chains converts the internal flavor states to flavor waves described by the Heisenberg Hamiltonian. Section 6 closes the paper with a preliminary discussion of string interactions together with some concluding remarks.

\section{Superstring bit models}

The type IIB superstring theory [20-27] quantized on the lightcone [12-15] is based on a worldsheet system with 8 transverse coordinates $x^{k}(\sigma, \tau)$, and 8 left moving and 8 right moving Grassmann variables $\theta_{L, R}^{a}(\sigma, \tau)$. We contemplate a more general worldsheet system with $d$ coordinates and $s$ pairs of Grassmann variables. The values $d=s=8$ are necessary for Poincaré supersymmetry, but the general case, which lacks these symmetries, is a perfectly sensible dynamical system worthy of study in its own right. Even though full Poincaré invariance is lost when $s \neq 8$ and/or $d \neq 8$, the lightcone dynamics still naturally implements a relativistic energy momentum dispersion law $P^{-}=\left(\boldsymbol{p}^{2}+\mu^{2}\right) / 2 P^{+}$in the 't Hooft limit, so the concept of particle mass is retained at least in the limit $N \rightarrow \infty$. In particular, it was emphasized in [9] that when $s>d$ there is a gap (i.e. the lowest $\mu^{2}>0$ ) in the mass spectrum of the string system, which tames infrared divergences. This gap vanishes for $d=s$ and is tachyonic $\mu^{2}<0$, leading to instabilities, when $d>s$. Because the closed chains are noninteracting when $N=\infty$, these instabilities are problematic only at finite $N$.

As mentioned in the introduction we set up superstring bit dynamics in the standard second-quantized formalism, with creation and annihilation operators for a string bit. In general, a superstring bit annihilation operator is an $N \times N$ matrix denoted by

$$
\left(\phi_{\left[a_{1} \cdots a_{n}\right]}^{f_{1} \cdots f_{d}}\right)_{\alpha}^{\beta}, \quad n=0, \ldots, s ; \quad a_{j}=1, \ldots, s ; \quad \alpha, \beta=1, \ldots, N
$$

The $f_{j}$ are "flavor" indices, describing the degrees of freedom responsible for the emergence of transverse space. The $a_{k}$ are spinor indices, with the square brackets enclosing them reminding us that $\phi$ is completely antisymmetric under permutations of them. Also $\phi$ will be bosonic (fermionic) if the number of spinor indices $n$ is even (odd). We will denote the corresponding creation operator by $\bar{\phi}_{\alpha}^{\beta} \equiv\left(\phi_{\beta}^{\alpha}\right)^{\dagger}$. In the simplest realization of $d$ space coordinates proposed in [9], it is enough that each $f_{j}=1,2$. In that model for the 
superstring, the Hamiltonian is

$$
H=H_{F}+H_{S}
$$

where the flavor dynamics is given by

$$
H_{F}=\frac{2}{N} \sum_{n=0}^{s} \sum_{k=0}^{s} \frac{1}{n ! k !} \operatorname{Tr} \bar{\phi}_{a_{1} \cdots a_{n}}^{E} \bar{\phi}_{b_{1} \cdots b_{k}}^{F} \phi_{b_{1} \cdots b_{k}}^{G} \phi_{a_{1} \cdots a_{n}}^{H} V_{E F G H}
$$

In this formula each capital superscript represents the collection of the individual flavor indices $F=\left\{f_{j}\right\}$. The spinor dynamics is described by

$$
H_{S}=H_{1}+H_{2}+H_{3}+H_{4}+H_{5}
$$

where the $H_{i}$ are:

$$
\begin{aligned}
& H_{1}=\frac{2}{N} \sum_{n=0}^{s} \sum_{k=0}^{s} \frac{s-2 n}{n ! k !} \operatorname{Tr} \bar{\phi}_{a_{1} \cdots a_{n}}^{F} \bar{\phi}_{b_{1} \cdots b_{k}}^{G} \phi_{b_{1} \cdots b_{k}}^{G} \phi_{a_{1} \cdots a_{n}}^{F} \\
& H_{2}=\frac{2}{N} \sum_{n=0}^{s-1} \sum_{k=0}^{s-1} \frac{(-)^{k}}{n ! k !} \operatorname{Tr} \bar{\phi}_{a_{1} \cdots a_{n}}^{F} \bar{\phi}_{b b_{1} \cdots b_{k}}^{G} \phi_{b_{1} \cdots b_{k}}^{G} \phi_{b a_{1} \cdots a_{n}}^{F} \\
& H_{3}=\frac{2}{N} \sum_{n=0}^{s-1} \sum_{k=0}^{s-1} \frac{(-)^{k}}{n ! k !} \operatorname{Tr} \bar{\phi}_{b a_{1} \cdots a_{n}}^{F} \bar{\phi}_{b_{1} \cdots b_{k}}^{G} \phi_{b b_{1} \cdots b_{k}}^{G} \phi_{a_{1} \cdots a_{n}}^{F} \\
& H_{4}=\frac{2 i}{N} \sum_{n=0}^{s-1} \sum_{k=0}^{s-1} \frac{(-)^{k}}{n ! k !} \operatorname{Tr} \bar{\phi}_{a_{1} \cdots a_{n}}^{F} \bar{\phi}_{b_{1} \cdots b_{k}}^{G} \phi_{b b_{1} \cdots b_{k}}^{G} \phi_{b a_{1} \cdots a_{n}}^{F} \\
& H_{5}=-\frac{2 i}{N} \sum_{n=0}^{s-1} \sum_{k=0}^{s-1} \frac{(-)^{k}}{n ! k !} \operatorname{Tr} \bar{\phi}_{b a_{1} \cdots a_{n}}^{F} \bar{\phi}_{b b_{1} \cdots b_{k}}^{G} \phi_{b_{1} \cdots b_{k}}^{G} \phi_{a_{1} \cdots a_{n}}^{F}
\end{aligned}
$$

The formula for $H_{S}$ can be inferred from the superstring bit Hamiltonian proposed in $[2,3]$ by discarding all contributions from the transverse coordinates. The structure of $H$ is designed so that the action of $H$ on single trace states goes, in the 't Hooft limit $N \rightarrow$ $\infty[4,5]$, to the action of a discretized version of the first quantized Hamiltonian for the worldsheet fields of the type IIB superstring.

To explain this we introduce $s$ Grassmann variables $\theta^{a}$ and construct the super creation operators

$$
\psi^{F}(\theta)=\sum_{k=0}^{s} \frac{1}{k !} \bar{\phi}_{c_{1} \cdots c_{k}}^{F} \theta^{c_{1}} \cdots \theta^{c_{k}}
$$

so that a general single trace state can be constructed from

$$
\left|\theta_{1} F_{1}, \cdots, \theta_{M} F_{M}\right\rangle=\operatorname{Tr} \psi^{F_{1}}\left(\theta_{1}\right) \cdots \psi^{F_{M}}\left(\theta_{M}\right)|0\rangle
$$

We note that the cyclic property of the trace implies the cyclic symmetry condition

$$
\left|\theta_{1} F_{1}, \cdots, \theta_{M} F_{M}\right\rangle=\left|\theta_{2} F_{2}, \cdots, \theta_{M} F_{M}, \theta_{1} F_{1}\right\rangle
$$


Then we evaluate

$$
\begin{aligned}
& H_{F}\left|\theta_{1} F_{1}, \cdots, \theta_{M} F_{M}\right\rangle=2 \sum_{k=1}^{M}\left|\theta_{1} F_{1}, \cdots, \theta_{k} G, \theta_{k+1} G^{\prime}, \cdots, \theta_{M} F_{M}\right\rangle V_{G G^{\prime} F_{k+1} F_{k}}+\mathcal{O}\left(N^{-1}\right) \\
& H_{1}\left|\theta_{1} F_{1}, \cdots, \theta_{M} F_{M}\right\rangle=2 \sum_{k=1}^{M}\left(s-2 \theta_{k}^{a} \frac{d}{d \theta_{k}^{a}}\right)\left|\theta_{1} F_{1}, \cdots, \theta_{M} F_{M}\right\rangle+\mathcal{O}\left(N^{-1}\right) \\
& H_{2}\left|\theta_{1} F_{1}, \cdots, \theta_{M} F_{M}\right\rangle=2 \sum_{k=1}^{M} \theta_{k}^{a} \frac{d}{d \theta_{k+1}^{a}}\left|\theta_{1} F_{1}, \cdots, \theta_{M} F_{M}\right\rangle+\mathcal{O}\left(N^{-1}\right) \\
& H_{3}\left|\theta_{1} F_{1}, \cdots, \theta_{M} F_{M}\right\rangle=2 \sum_{k=1}^{M} \theta_{k+1}^{a} \frac{d}{d \theta_{k}^{a}}\left|\theta_{1} F_{1}, \cdots, \theta_{M} F_{M}\right\rangle+\mathcal{O}\left(N^{-1}\right) \\
& H_{4}\left|\theta_{1} F_{1}, \cdots, \theta_{M} F_{M}\right\rangle=-2 i \sum_{k=1}^{M} \theta_{k}^{a} \theta_{k+1}^{a}\left|\theta_{1} F_{1}, \cdots, \theta_{M} F_{M}\right\rangle+\mathcal{O}\left(N^{-1}\right) \\
& H_{5}\left|\theta_{1} F_{1}, \cdots, \theta_{M} F_{M}\right\rangle=-2 i \sum_{k=1}^{M} \frac{d}{d \theta_{k}^{a}} \frac{d}{d \theta_{k+1}^{a}}\left|\theta_{1} F_{1}, \cdots, \theta_{M} F_{M}\right\rangle+\mathcal{O}\left(N^{-1}\right)
\end{aligned}
$$

To formulate the energy spectrum problem at $N=\infty$ in the first quantized language we express the sought eigenstate as

$$
|E\rangle=\int d^{s} \theta_{1} \cdots d^{s} \theta_{M}\left|\theta_{1} F_{1}, \cdots, \theta_{M} F_{M}\right\rangle \Psi\left(\theta_{1}, \ldots, \theta_{M}\right) U_{F_{1} \cdots F_{M}} .
$$

Because of the cyclic property (2.12), we can, without loss of generality, require the wave function to satisfy

$$
\Psi\left(\theta_{1}, \ldots, \theta_{M}\right) U_{F_{1} \cdots F_{M}}=(-)^{s(M-1)} \Psi\left(\theta_{2}, \ldots, \theta_{M}, \theta_{1}\right) U_{F_{2} \cdots F_{M} F_{1}},
$$

where the sign out front arises from reordering the $d^{s} \theta_{k}$ 's. The wave functions must be anticyclic if both $s$ and $M-1$ are odd, and cyclic otherwise.

We now apply $H$ to $|E\rangle$ and write out the eigenvalue condition:

$$
E|E\rangle=H|E\rangle=\int d^{s} \theta_{1} \cdots d^{s} \theta_{M}\left|\theta_{1} F_{1}, \cdots, \theta_{M} F_{M}\right\rangle h\left(\Psi\left(\theta_{1}, \ldots, \theta_{M}\right) U_{F_{1} \cdots F_{M}}\right)
$$

for $N=\infty$. An integration by parts in the $\theta$ variables is done in the last step, after which one finds $h=h_{S}+h_{F}$ with

$$
h_{S}=2 \sum_{k=1}^{M}\left[-i \theta_{k}^{a} \theta_{k+1}^{a}-i \frac{d}{d \theta_{k}^{a}} \frac{d}{d \theta_{k+1}^{a}}-\theta_{k}^{a} \frac{d}{d \theta_{k+1}^{a}}-\theta_{k+1}^{a} \frac{d}{d \theta_{k}^{a}}-s+2 \theta_{k}^{a} \frac{d}{d \theta_{k}^{a}}\right],
$$

and $h_{F}$ acts as a matrix

$$
\left\langle G_{1} \cdots G_{M}\left|h_{F}\right| F_{1} \cdots F_{M}\right\rangle=\sum_{k=1}^{M} \delta_{G_{1}}^{F_{1}} \cdots \delta_{G_{k-1}}^{F_{k-1}} V_{G_{k} G_{k+1} F_{k+1} F_{k}} \delta_{G_{k+2}}^{F_{k+2}} \cdots \delta_{G_{M}}^{F_{M}}
$$


In other words $h_{F}$ is a sum of $M$ terms, where the $k$ th term acts as the identity on all the indices of $U$ except for $F_{k}$ and $F_{k+1}$, and acts on these two as a matrix. As a useful shorthand in the first quantized dynamics we can write

$$
\begin{aligned}
h_{F} & =\sum_{k=1}^{M} V^{k} \\
\left\langle G_{1} \cdots G_{M}\left|V^{k}\right| F_{1} \cdots F_{M}\right\rangle & =\delta_{G_{1}}^{F_{1}} \cdots \delta_{G_{k-1}}^{F_{k-1}} V_{G_{k} G_{k+1} F_{k+1} F_{k}} \delta_{G_{k+2}}^{F_{k+2}} \cdots \delta_{G_{M}}^{F_{M}}
\end{aligned}
$$

This nearest neighbor structure of $h_{F}$, presents the flavor dynamics, of single trace states of the string bit system in 't Hooft's limit $N \rightarrow \infty$, as a generalized one dimensional chain. The size of the chain $M$ is just the number of bits in the single trace state.

The specifics of the chain dynamics are controlled by the coefficients $V_{E F G H}$ appearing in $H_{F}$ (2.3). We have introduced these flavor degrees of freedom in order to produce the transverse spatial dimensions of the lightcone string. It is well-known that the low energy states of a Heisenberg spin chain with $M$ spins behave at large $M$ like those of a coordinate compactified to a circle. Moreover, this chain model is flexible enough to give a continuously variable compactification circumference $L$, including the limit $L \rightarrow \infty$. In the rest of this paper, for definiteness, we choose the $V_{E F G H}$ so that the $N \rightarrow \infty$ limit implies that $h_{F}$ is the sum of $d$ independent Heisenberg spin chain Hamiltonians:

$$
h_{F}=\sum_{l=1}^{d} C_{l} \sum_{k=1}^{M}\left[\left(\sigma_{l}\right)_{k}^{1}\left(\sigma_{l}\right)_{k+1}^{1}+\left(\sigma_{l}\right)_{k}^{2}\left(\sigma_{l}\right)_{k+1}^{2}+\Delta_{l}\left(\sigma_{l}\right)_{k}^{3}\left(\sigma_{l}\right)_{k+1}^{3}\right]
$$

Here, for each $l, k,\left(\sigma_{l}\right)_{k}^{1,2,3}$ are the two by two Pauli spin matrices

$$
\sigma^{1}=\left(\begin{array}{ll}
0 & 1 \\
1 & 0
\end{array}\right), \quad \sigma^{2}=\left(\begin{array}{cc}
0 & -i \\
i & 0
\end{array}\right), \quad \sigma^{3}=\left(\begin{array}{cc}
1 & 0 \\
0 & -1
\end{array}\right)
$$

That is, the first quantized Hamiltonian $h_{F}$ is the sum of $d$ commuting operators, each the Hamiltonian of a Heisenberg spin system. This choice is certainly not unique. For one thing it commits the outcome to toroidal compactification of the transverse coordinates. There are presumably other chain systems that share this feature. And we can expect more sophisticated choices to produce more interesting compact manifolds. It is important in our world, of course, that at least two transverse coordinate dimensions are very large if not infinite. But any extra dimensions should reside in some compact manifold, which our choice limits to a torus.

Studies by Bethe [17] and Yang and Yang [18, 19], which we review in section 4 and the appendix, enable the exact calculation of the low lying energy spectrum of the spin chain when $M \rightarrow \infty$. As explained in [28] these energy eigenstates are identical to those of a spatial coordinate compactified on a circle. In addition to string vibrational modes, there are two zero modes, one corresponding to the string momentum and the other to winding the string around the compactification circle. The radius of the circle is related to the parameter $\Delta_{l}$, so each dimension can be compactified on a different sized circle. The 
infinite radius limit, corresponding to a noncompact spatial dimension, is reached by the limit $\Delta_{l} \rightarrow 1$, if $C_{l}<0$ and $\Delta_{l} \rightarrow-1$ if $C_{l}>0$.

Our choice then determines the coefficients $V_{G G^{\prime} F^{\prime} F}$ of the string bit Hamiltonian. Each capital index is a string of two-valued indices $F=\left\{f_{1} \cdots f_{d}\right\}$ and similarly for $G, F^{\prime}, G^{\prime}$. Then

$$
V_{G G^{\prime} F^{\prime} F}=\sum_{l=1}^{d} C_{l}\left[\sigma_{g_{l} f_{l}}^{1} \sigma_{g_{l}^{\prime} f_{l}^{\prime}}^{1}+\sigma_{g_{l} f_{l}}^{2} \sigma_{g_{l}^{\prime} f_{l}^{\prime}}^{2}+\Delta_{l} \sigma_{g_{l} f_{l}}^{3} \sigma_{g_{l}^{\prime} f_{l}^{\prime}}^{3}\right] \prod_{k \neq l}\left(\delta_{g_{k} f_{k}} \delta_{g_{k}^{\prime} f_{k}^{\prime}}\right) .
$$

When there is no danger of confusion, we will suppress the indices on the right side of this equation which we can write:

$$
V_{G G^{\prime} F^{\prime} F} \rightarrow \sum_{l=1}^{d} C_{l}\left[\sigma_{l}^{1} \sigma_{l}^{1 \prime}+\sigma_{l}^{2} \sigma_{l}^{2 \prime}+\Delta_{l} \sigma_{l}^{3} \sigma_{l}^{3 \prime}\right]
$$

In this notation the $\sigma_{l}^{1,2,3}, \sigma_{l}^{\prime 1,2,3}$ commute with the $\sigma_{l^{\prime}}^{1,2,3}, \sigma_{l^{\prime}}^{\prime 1,2,3}$ when $l \neq l^{\prime}$ and the $\sigma_{l}^{1,2,3}$ commute with the $\sigma_{l}^{\prime 1,2,3}$.

\section{Emergence of Grassmann and longitudinal space}

At finite $N$ the string bit system has a finite number of degrees of freedom: for our example this number is $2^{s+d} N^{2}$. In the large $N$ limit the low energy eigenstates with a large bit number $M$ show an energy excitation of order $1 / M$. Recalling that the lightcone mass shell condition for a particle is $P^{-}=\left(m^{2}+\boldsymbol{p}^{2}\right) / 2 P^{+}$, we seek to interpret $M$ as $P^{+}$and $H$ as $P^{-}$. The eigenvalues of $h_{S}$ at fixed $M$ are $[2,3,9]$

$$
\begin{aligned}
E_{S} & =E_{S}^{\min }+8 \sum_{n=1}^{M-1} \eta_{n} \sin \frac{n \pi}{M}, \quad \eta_{n}=0,1, \ldots, s \\
E_{S}^{\min } & =-4 s \cot \frac{\pi}{2 M} \sim-\frac{s 8 M}{\pi}+\frac{2 \pi s}{3 M}
\end{aligned}
$$

corresponding to states built from fermionic creation operators $B_{n}^{a \dagger}, n=0, \ldots, M-1$, applied to a ground state $|0\rangle$. Here the modes $n$ and $M-n$ have the same frequency. Excitations of order $1 / M$ arise when $M \rightarrow \infty$ with either $n / M \ll 1$ or $(M-n) / M \ll 1$. We can call the first case left moving modes and the second right moving modes. When $s$ is even, the cyclic symmetry constraint amounts to the requirement that $N_{L}=N_{R}$ where $N_{L}$, $N_{R}$ are the total mode numbers of left moving right moving modes respectively. The zero mode $B_{0}$ converts boson states to fermion states and vice versa. Its existence establishes that the number of bosonic states is the same as the number of fermionic states.

When $s=8$ this excitation spectrum is precisely that of the left and right moving Grassmann worldsheet fields $\theta_{L}^{a}, \theta_{R}^{a}$ of the Green-Schwarz formulation of the type IIB superstring. This identification fixes the scale of the energy in terms of $m$ the unit of $P^{+} \equiv M m$ and the rest tension of the string $T_{0}=1 /\left(2 \pi \alpha^{\prime}\right)$. Each worldsheet coordinate field should contribute $-\pi T_{0} /\left(6 P^{+}\right)$to the closed string ground state $P^{-}$. To see this just 
consider the bosonic string which has 24 such coordinate worldsheet fields. We know that the ground state mass squared of the bosonic closed string is $2 P^{+} P^{-}=-4 / \alpha^{\prime}=-8 \pi T_{0}$. For the superstring each left-right pair of Grassmann fields contribute just the negative of this, namely $\pi T_{0} /\left(6 P^{+}\right)$. Remembering that $P^{+}=M m$, it follows that $P_{S}^{-}=E_{S} T_{0} /(4 m)$, or for operators

$$
\begin{aligned}
& P^{-}=P_{S}^{-}+P_{F}^{-} \\
& P_{S}^{-}=\frac{T_{0}}{4 m}\left(H_{1}+H_{2}+H_{3}+H_{4}+H_{5}\right)
\end{aligned}
$$

and $P_{F}^{-} \equiv\left(T_{0} / 4 m\right) H_{F}$ will be determined after the analysis of the next section.

\section{Heisenberg spin chain}

The conventional Hamiltonian for the spin chain is usually defined as

$$
H_{h e i}=-\sum_{k=1}^{M}\left(\sigma_{k}^{1} \sigma_{k+1}^{1}+\sigma_{k}^{2} \sigma_{k+1}^{2}+\Delta \sigma_{k}^{3} \sigma_{k+1}^{3}\right)
$$

The application to string bit dynamics requires periodic boundary conditions, meaning $\sigma_{M+1} \equiv \sigma_{1}$, which we henceforth assume. It is well-known that critical behavior is present for the range $-1 \leq \Delta \leq 1$. The minus sign out front nominally favors spin alignment, but for $\Delta$ in this range the lowest energy states actually have charge (spin) $Q=\sum_{k} \sigma_{k}^{3}=0$. The solution for the eigenvalues of $H$ is given by the Bethe ansatz [17], in which eigenstates are sought as spin waves of overturned spins relative to the state $|0\rangle$, in which $\sigma_{k}^{3}=+1$ for all $k$. Then a state with $q$ overturned spins is denoted $\left|i_{1}, \cdots, i_{q}\right\rangle$ where the $i_{k}$ give the locations of the overturned spins. Then the Bethe ansatz is

$$
\begin{aligned}
|E\rangle & =\sum_{P} A_{P}\left|i_{1}, \cdots, i_{q}\right\rangle e^{i \sum_{k} i_{k} p_{P_{k}}} \\
H|0\rangle & =-M \Delta|0\rangle, \quad E=-M \Delta+4 \sum_{k=1}^{q}\left(\Delta-\cos p_{k}\right) .
\end{aligned}
$$

Here, the sum over $P$ is the sum of all permutations of $12 \cdots q$. Without loss of generality, we may restrict $-\pi \leq p_{k} \leq \pi$. The ansatz is an eigenstate of $H$, provided that the $p_{k}$, which must all be distinct, satisfy

$$
\begin{aligned}
p_{k} & =\frac{2 \pi I_{k}}{M}-\frac{1}{M} \sum_{j=1}^{q} \theta\left(p_{k}, p_{j}\right) \\
\theta\left(p_{k}, p_{j}\right) & \equiv 2 \arctan \frac{\left.\Delta \sin \left(\left(p_{k}-p_{j}\right) / 2\right)\right)}{\cos \left(\left(p_{k}+p_{j}\right) / 2\right)-\Delta \cos \left(\left(p_{k}-p_{j}\right) / 2\right)}
\end{aligned}
$$

Periodic boundary conditions require that the $I_{k}$ are integers when $q$ is odd, or that they are half odd integers when $q$ is even. We can identify two conserved quantities that help characterize the different eigenstates especially at large $M$. One is the total charge $Q=$ 
$M-2 q$ for the state with $q$ overturned spins. The other is the total momentum of the overturned spins

$$
P=\sum_{k} p_{k}=\frac{2 \pi}{M} \sum_{k} I_{k}
$$

The last equality follows from the antisymmetry of $\theta\left(k, k^{\prime}\right)=-\theta\left(k^{\prime}, k\right)$. Inspection of the form of the energy eigenvalue shows that the energy is minimized (maximized) for the maximum value of $q$ for which all of the overturned spins satisfy $\Delta-\cos p_{k}<0(>0)$.

\subsection{Energy analysis for $\Delta=0$}

The equation (4.4) is formidable but Yang and Yang have successfully analyzed it for large $M[18,19]$. We review their analysis in the appendix. Here we discuss the case $\Delta=0$ for which $\theta=0$, and hence $p_{k}=2 \pi I_{k} / M$ exactly for all $M$. Focusing first on lowest energy states, we are interested in all the $p_{k}$ in the range $-\pi / 2<p_{k}<\pi / 2$. When $q$ is even (odd) the $p_{k}$ are of the form $\pi\left(2 n_{k}+1\right) / M\left(2 \pi n_{k} / M\right)$. For fixed $M, q$ the energy is minimized when the $n_{k}$ are consecutive integers as symmetrical about 0 as possible. So let the $n_{k}$ range from $n_{1}$ to $n_{2}$ spaced by integers. Then $q=n_{2}-n_{1}+1$, and

$$
E= \begin{cases}-4 \sum_{k=n_{1}}^{n_{2}} \cos \frac{2 \pi k}{M}=-2 \frac{\sin \left[\pi\left(2 n_{2}+1\right) / M\right]-\sin \left[\pi\left(2 n_{1}-1\right) / M\right]}{\sin [\pi / M]}, & q \quad \text { odd } \\ -4 \sum_{k=n_{1}}^{n_{2}} \cos \frac{\pi(2 k+1)}{M}=-2 \frac{\sin \left[2 \pi\left(n_{2}+1\right) / M\right]-\sin \left[2 \pi n_{1} / M\right]}{\sin [\pi / M]}, & q \text { even }\end{cases}
$$

For the given $M$, find an integer $r$ such that $M-r$ is divisible by 4 . We may take $r$ from the set $\{-1,0,1,2\}$. Then define $l, k$ by $q=(M-r) / 2+l$ and $n_{1}=-(M-r) / 4+k$. It follows that $Q=r-2 l, n_{2}=l+k-1+(M-r) / 4$. Note that $q$ is even (odd) if and only if $l$ is even (odd). Plugging these expressions into the energy formulas,

$$
E= \begin{cases}-2 \frac{\cos [\pi(-Q+2 k-1+r / 2) / M]+\cos [\pi(2 k-1+r / 2) / M]}{\sin [\pi / M]}, & l \text { odd } \\ -2 \frac{\cos [\pi(-Q+2 k+r / 2) / M]+\cos [\pi(2 k+r / 2) / M]}{\sin [\pi / M]}, & l \text { even }\end{cases}
$$

In this form we can take the long chain limit $M \rightarrow \infty$ with $l, k$ fixed, and identify the low-lying excitations.

$$
E \sim \begin{cases}4\left[-\frac{M}{\pi}-\frac{\pi}{6 M}+\frac{\pi}{M}\left(\frac{Q^{2}}{8}+2\left\{k+\frac{l-1}{2}\right\}^{2}\right)\right], & l \text { odd } \\ 4\left[-\frac{M}{\pi}-\frac{\pi}{6 M}+\frac{\pi}{M}\left(\frac{Q^{2}}{8}+2\left\{k+\frac{l}{2}\right\}^{2}\right)\right], & l \text { even }\end{cases}
$$

Note that whether $l$ is even or odd, the quantity in braces is any integer. Also, $Q$ is an even (odd) integer if $M$ is even (odd). This quantity has a simple interpretation in terms 
of the total momentum $P$ :

$$
P= \begin{cases}\sum_{k=n_{1}}^{n_{2}} \frac{2 \pi k}{M}=\frac{\pi}{M}(l+2 k-1) \frac{M+2 l-r}{2} \sim \pi\left(k+\frac{l-1}{2}\right) & l \text { odd } \\ \sum_{k=n_{1}}^{n_{2}} \frac{\pi(2 k+1)}{M}=\frac{\pi}{M}(l+2 k) \frac{M+2 l-r}{2} \sim \pi\left(k+\frac{l}{2}\right) & l \text { even }\end{cases}
$$

where the last forms are for $M \rightarrow \infty$. Thus we can write the final answer

$$
E \sim 4\left[-\frac{M}{\pi}-\frac{\pi}{6 M}+\frac{\pi}{M}\left(\frac{Q^{2}}{8}+2 \frac{P^{2}}{\pi^{2}}\right)\right]
$$

where $Q$ and $P / \pi$ can be any pair of integers, with the evenness or oddness of $Q$ correlated with that of $M$.

The energy spectrum for $\Delta \neq 0$ involves the full sophistication of the Bethe ansatz. Once it is fully implemented, one can take the large $M$ limit to find the low lying energy spectrum of $H(\Delta)$. The analysis of $[18,19]$, reviewed in the appendix, shows that the only effect of $\Delta \neq 0$ is to alter the overall constant out front and the coefficients of $M, Q^{2}$ and $P^{2}$ :

$$
\begin{aligned}
& E \sim \frac{2 \pi \sin \mu}{\mu}\left[-\alpha(\mu) \frac{M}{\pi}-\frac{\pi}{6 M}+\frac{\pi}{M}\left(\frac{\pi-\mu}{\pi} \frac{Q^{2}}{4}+\frac{\pi}{\pi-\mu} \frac{P^{2}}{\pi^{2}}+2\left(N_{L}+N_{R}\right)\right)\right] \\
& \Delta=-\cos \mu
\end{aligned}
$$

The term involving $\left(N_{L}+N_{R}\right)$ gives the spin wave excitations, which correspond to particlehole configurations of the momenta near the "Fermi sea" of the overturned spins. The $-\pi /(6 M)$ term is the universal part of the zero point energy associated with these particlehole excitations [29].

\subsection{Lowest lying energies of $H^{\prime}=-H_{\mathrm{Hei}}$}

An interesting twist occurs if the Hamiltonian is taken to be $-H$. Then the lowest energy states correspond to the highest energy eigenstates of $H$. To find these for $\Delta=0$, we now require that the $p_{k}$ all satisfy $\cos p_{k}<0$. Thus $\pi / 2<p_{k} \leq \pi$ or $-\pi<p_{k}<-\pi / 2$. Adding $2 \pi$ to the $p_{k}$ in the second category allows the two categories to be unified to $\pi / 2<p_{k}<3 \pi / 2$. So now we define $k, l$ by $n_{1}=k+(M-r) / 4, q=l+(M-r) / 2$, $n_{2}=k+l-1+3(M-r) / 4$. Using these definitions the energy can be written

$$
\begin{aligned}
& E= \begin{cases}2 \frac{\cos [\pi(2 k+2 l-1-3 r / 2) / M]+\cos [\pi(2 k-1-r / 2) / M]}{\sin [\pi / M]}, & l \quad \text { odd } \\
2 \frac{\cos [\pi(2 k+2 l-3 r / 2) / M]+\cos [\pi(2 k+(M-r) / 2) / M]}{\sin [\pi / M]}, & l \text { even }\end{cases} \\
& E \sim \begin{cases}4\left[\frac{M}{\pi}+\frac{\pi}{6 M}-\frac{\pi}{M}\left(\frac{Q^{2}}{8}+2\left\{k+\frac{l-1-r}{2}\right\}^{2}\right)\right], & l \text { odd } \\
4\left[\frac{M}{\pi}+\frac{\pi}{6 M}-\frac{\pi}{M}\left(\frac{Q^{2}}{8}+2\left\{k+\frac{l-r}{2}\right\}^{2}\right)\right], & l \text { even }\end{cases}
\end{aligned}
$$


where the second line shows the large $M$ behavior. In this case the quantities in braces are integers when $r$ (and also $M$ ) are even and half odd integers when $r$ (and also $M$ ) are odd. In the latter odd case, $Q$ is also odd, with the implication that when $M$ is odd neither of the squared terms can ever be zero.

With the $p_{k}$ in the range $\pi / 2<p_{k}<3 \pi / 2$, as we have chosen here, the total momentum works out to:

$$
P= \begin{cases}\frac{\pi}{M}(M-r+l+2 k-1) \frac{M+2 l-r}{2} \sim q \pi+\pi\left(k+\frac{l-1-r}{2}\right) & l \text { odd } \\ \frac{\pi}{M}(M-r+l+2 k) \frac{M+2 l-r}{2} \sim q \pi+\pi\left(k+\frac{l-r}{2}\right) & l \text { even }\end{cases}
$$

where in the last forms, we have dropped some terms of order $M^{-1}$. We see that the quantity $(P-q \pi) / \pi$ approaches the quantities in braces, so that we can write the highest energies as

$$
E \sim 4\left[\frac{M}{\pi}+\frac{\pi}{6 M}-\frac{\pi}{M}\left(\frac{Q^{2}}{8}+2 \frac{(P-q \pi)^{2}}{\pi^{2}}\right)\right]
$$

As we have mentioned the high energy spectrum of $H$ becomes the low energy spectrum of $-H$. When $M$ is even these two Hamiltonians are similar. We have just found that when $M$ is odd, the energy spectrum of $-H(0)$ is not the same as $H(0)$. This implies that the two Hamiltonians are not similar when $M$ is odd.

For $\Delta \neq 0$, the highest eigenvalues of $H$ are still the lowest eigenvalues of $-H_{\mathrm{Hei}}$ :

$$
H^{\prime}=-H_{\mathrm{Hei}}(\Delta)=\sum_{k=1}^{M}\left(\sigma_{k}^{1} \sigma_{k+1}^{1}+\sigma_{k}^{2} \sigma_{k+1}^{2}+\Delta \sigma_{k}^{3} \sigma_{k+1}^{3}\right) \text {. }
$$

When $M$ is even, the operator $C=\prod_{k=\text { odd }} \sigma_{k}^{3}=C^{-1}=C^{\dagger}$ relates $H^{\prime}$ to the original Heisenberg Hamiltonian with $\Delta \rightarrow-\Delta$.

$$
H^{\prime}=C H_{\mathrm{Hei}}(-\Delta) C
$$

It immediately follows that the lowest energy eigenvalues of $H^{\prime}$ are those of $H_{\mathrm{Hei}}$ with $\mu \rightarrow \pi-\mu$ :

$$
E^{\prime}-E_{0}^{\prime}=\frac{2 \pi \sin \mu}{\pi-\mu}\left[-\frac{\pi}{6}+\frac{\mu}{4} Q^{2}+\frac{1}{\mu} \hat{P}^{2}+2 \pi\left(N_{R}+N_{L}\right)\right] \frac{1}{M}, \quad M \quad \text { even }
$$

Here $\hat{P}$ is the total momentum in the Bethe ansatz for an eigenstate $\left|\left\{p_{k}\right\}\right\rangle$ of $H_{\text {Hei }}(-\Delta)$. The corresponding eigenstate of $H^{\prime}$ is $C\left|\left\{p_{k}\right\}\right\rangle$. The operator $C$ multiplies each term, in the Bethe ansatz with an odd number of overturned spins sitting on odd sites, by -1 . But this is equivalent to adding $\pi$ to each of the $p_{k}$. Indeed, it is easy to show that if the $p_{k}$ satisfy the Bethe ansatz conditions for $-\Delta$, then $p_{k}+\pi$ satisfy the Bethe ansatz conditions for $\Delta$. Thus the total momentum in the Bethe ansatz for $H^{\prime}$ is $P=\hat{P}+q \pi$. thus we can write

$$
E^{\prime}-E_{0}^{\prime}=\frac{2 \pi \sin \mu}{\pi-\mu}\left[-\frac{\pi}{6}+\frac{\mu}{4} Q^{2}+\frac{1}{\mu}(P-q \pi)^{2}+2 \pi\left(N_{R}+N_{L}\right)\right] \frac{1}{M} .
$$


We remind the reader that we have been assuming $M$ is even, to rigorously obtain these results without additional work. However the continuum analysis that leads directly to all these results is quite insensitive to the evenness of oddness of $M$. Hence the restriction to even $M$ can be dropped. The discrete nature of $Q$ and $P$ is sensitive to the parity of $M$, but not to the value of $\Delta$. Thus $Q=M-2 q$ is even (odd) if $M$ is even (odd). Similarly $P=(2 \pi / M) \sum_{k} I_{k}$ assumes for all $\Delta$ the values it has for $\Delta=0$. In the low lying states of $H_{\mathrm{Hei}}$ these are integer multiples of $\pi$ for all $M$, even and odd. But in the high lying states of $H_{\text {Hei }}$ (low lying states of $H^{\prime}$ ), the values of $P-q \pi$ are integer multiples of $\pi$ for even $M$, but half odd integer multiples of $\pi$ for odd $M$.

\section{$5 \quad$ Emergence of transverse space}

In the previous section and in the appendix we have sketched the analysis of the low energy states of the Heisenberg spin chain. A word of explanation for why this suffices for our purposes. Each string bit carries a unit $m$ of $P^{+}$. A single trace state of $M$ string bits, which in the large $N$ limit will describe a free single string, carries a total $P^{+}=M m$. $M$ must be large for $P^{+}$to be treated as a continuous variable. Furthermore, the energy of the system, which is to be identified with $P^{-}$, must, for the continuous string, scale as $1 / P^{+}=1 /(M m)$. This means that for fixed parameters, the only important excitations of $h_{F}$ are those with energy of order $1 / M$ above the ground energy, and for large $M$ these are the low lying energy states correctly described by our analysis. It should be remembered that such $1 / M$ excitations cannot occur unless the model is critical. This is why we require $-1 \leq \Delta \leq 1$ ! The Heisenberg chain (and related vertex models) are rather special in having a line rather than discrete points of critical behavior. Higher lying states actually have energies that are bigger than these by a positive power of $M$ as $M$ gets large. This is simply because the parameters of the model Hamiltonian are independent of $M$. Of course for the ultimate stability of the whole scheme in the presence of interactions (i.e. at finite $N$ ), it should be true that a single string with $M$ bits have lower energy than two strings with $M_{1}$ and $M-M_{1}$ bits. This is a nontrivial requirement that fails, for example, for the string bit picture of the bosonic string. This issue is analyzed for a stable toy superstring bit model in [9]. The models discussed in the present paper are extensions of that toy model, and are expected to be stable, at least for large enough $N$.

We next obtain the parameters of $H_{F}$, for our chosen case where the flavor dynamics of the large $N$ limit is given by a collection of $d$ Heisenberg spin chains. We do this by matching the order $1 / M$ behavior of the eigenvalues of the $k$ th Heisenberg chain in $P_{F}^{-} \equiv\left(T_{0} / 4 m\right) H_{F}$, given by multiplying (4.12) or (4.21) by $T_{0}\left|C_{k}\right| /(4 m)$, to the eigenvalues of the lightcone Hamiltonian of the free positive tension fundamental string:

$$
P^{-}=\frac{1}{2} \int_{0}^{P^{+}} d \sigma\left[\mathcal{P}^{2}+T_{0}^{2} x^{\prime 2}\right]
$$

given by

$$
P^{-}=\frac{\pi T_{0}}{P^{+}}\left[-\frac{1}{6}+\frac{2 \pi}{L^{2} T_{0}} n^{2}+\frac{L^{2} T_{0}}{2 \pi} l^{2}+2\left(N_{L}+N_{R}\right)\right]
$$


where the coordinate $x$ lives on a circle of circumference $L$ and periodic boundary conditions under $x \rightarrow x+L$ are assumed. The momentum is $p=2 \pi n / L$. and $l$ is the number of times the closed string winds around the circle. In fundamental string theory, the circumference of the compactification circle need not be the same for each dimension. However, worldsheet conformal invariance (or closure of the Virasoro algebra) dictates that the level spacing given by the $N_{L}+N_{R}$ term be the same for each worldsheet degree of freedom, including the Grassmann degrees of freedom of the superstring already discussed in section 3 .

Matching the $N_{L}+N_{R}$ terms in $P_{F}^{-}$to those in (5.2) then gives, remembering that $P^{+}=M m$,

$$
C_{k}=-\frac{2 \mu_{k}}{\pi \sin \mu_{k}}
$$

in the $C_{k}<0$ case $(4.12)$ and

$$
C_{k}=\frac{2\left(\pi-\mu_{k}\right)}{\pi \sin \mu_{k}}
$$

in the $C_{k}>0$ case $(4.21)$.

With these values for the $C_{k}$, the $L_{k}$ dependent terms of (5.2) can be precisely matched to the corresponding terms in $P_{F}^{-}$, for chains with even bit number (and hence even $Q$ ). This matching then yields compactification circumferences $L_{k}$ determined by

$$
L_{k}^{2}=\frac{2 \pi}{T_{0}}\left(\frac{\pi}{\pi-\mu_{k}}\right) \quad \text { or } \quad \frac{2 \pi}{T_{0}}\left(\frac{\pi}{\mu_{k}}\right) .
$$

respectively. The chains with odd bit number (and hence odd $Q$ ) correspond in the string energy eigenvalues to half odd integer values of $n=k+1 / 2$ in (5.2) corresponding to plane wave functions $e^{i(2 k+1) x^{k} \pi / L_{k}}$ antiperiodic under $x^{k} \rightarrow x^{k}+L_{k}$. For $C_{k}<0$, the values of $l$ in (5.2) remain integral in the odd $M$ case. But for $C_{k}>0$ the $l$ associated with half odd $n$ in the odd $M$ case are also half odd integers. This has the interesting consequence that the decompactification limit $L_{k} \rightarrow \infty$ sends the odd bit number chains to infinite energy when $C_{k}>0$.

In our formulas, $T_{0}$ is a constant dimensionful parameter which sets the energy scale of the low lying excitations of long string bit chains. As such its actual value is nothing more than a choice of units. All that is important is that it is non-zero and positive. It is apparent that when $N=\infty$ the emergent space becomes non-compact in the limit $\mu_{k} \rightarrow \pi$ or $\mu_{k} \rightarrow 0$, in the respective cases. This is clearly a singular limit however, as is evident from the fact that the microscopic string bit parameters $C_{k}$ blow up in the limit. For this reason it is best to keep $\mu_{k}$ away from these limiting values as a handy infrared cutoff, when one studies the effects of interactions at finite $N$.

\section{Conclusion}

In this paper we have presented a class of stable string bit models in which space is an emergent phenomenon. The emphasis has been on the $N \rightarrow \infty$ limit of the models in which 
noninteracting strings form. Interactions will be present in these models at order $1 / N$. But although these interactions will be consistent with unitarity (by construction), they will not have the complexity to reproduce the interactions required of superstring theory.

By their very nature, $1 / N$ corrections can be interpreted as breaking and joining closed strings. This is because a noninteracting string state is a single trace state, and $1 / N$ corrections to the Hamiltonian acting on a multi-trace state either split one trace into two or join two traces into one. However as shown in [30] type IIB superstring theory requires that a rather complicated combination of coordinate and Grassmann fields be inserted at the joining point of the three string vertex. This insertion is quadratic in coordinate fields and an 8th order polynomial in Grassmann fields with the structure

$$
\mathcal{I}_{3}(\sigma)=\tilde{X}^{i}(\sigma) X^{j}(\sigma) v^{i j}(Y(\sigma))
$$

In this formula $\tilde{X}$ and $X$ are linear combinations of the worldsheet coordinate fields and $Y$ is a linear combination of the worldsheet Grassmann fields. The function $v^{i j}$ is a polynomial of $Y$ constructed from the following five monomials

$$
\delta_{i j}, \quad \gamma_{[a b}^{i k} \gamma_{c d]}^{j k} Y^{a} Y^{b} Y^{c} Y^{d}, \quad \delta_{i j} Y^{1} Y^{2} \cdots Y^{8}, \quad \gamma_{a b}^{i j} Y^{a} Y^{b}, \quad \gamma_{a b}^{i j} \epsilon^{a b c \cdots h} Y^{c} \cdots Y^{h}
$$

which transform as a 2-tensor in transverse space. Here $\gamma_{a b}^{i j}=\gamma_{a \dot{a}}^{i} \gamma_{b \dot{a}}^{j}-\delta_{i j} \delta_{a b}$, with $\gamma^{i}$ the $\mathrm{SO}(8)$ gamma matrices. The $1 / N$ corrections from the Hamiltonian presented in this paper can produce at most two factors of the Grassmann fields $Y$. To produce higher powers of $Y$, terms with more intricate spin structure must be added to the string bit Hamiltonian. In order to leave the large $N$ limit unaffected, we require these added terms to have the color structure

$$
\frac{g^{A B C D}}{N} \operatorname{Tr}: \bar{\phi}_{A} \phi_{B} \bar{\phi}_{C} \phi_{D}:
$$

where the $A, B, C, D$ signify the collection of spin and flavor indices carried by each $\phi$, and the colons indicate normal ordering. Such terms will not contribute in leading order in the $1 / N$ expansion. An important consequence is that the size of the coefficient $g^{A B C D}$ can be allowed to be very large with the perturbation still small, as long as $N \gg g^{A B C D}$. This flexibility will be particularly crucial if, as hinted by the analysis of [9], the stability of closed chains requires $M<N$. In that case, $N$ would have to be enormous to produce string-like chains. In that case the only relevant interactions would be those enhanced by a large coefficient.

It is a straightforward task to apply such terms to multi-trace states and determine the spinor index dependence of $g^{A B C D}$ which produces each of the monomials listed above. The flavor dependence responsible for the $X, \tilde{X}$ factors of the insertion is less obvious because the emergence of the effective coordinate fields from the Heisenberg chain dynamics is less direct than the emergence of the Grassmann fields. Effective coordinate fields only arise for large bit number chains. The construction of a string bit Hamiltonian which implies the fully interacting superstring in 10 spacetime dimensions remains a project for future research. 


\section{Acknowledgments}

I thank Oren Bergman, John Klauder, and Songge Sun for helpful discussions. This work is supported in part by U.S. DOE grant DE-FG02-97ER-41029.

\section{A Energy analysis for $-1<\Delta<1$, and large $M$}

Yang and Yang $[18,19]$ use the following techniques to solve the spin chain energies in the limit $M \rightarrow \infty$. They map the $p_{j}$ onto new variables $\alpha_{j}$ for which $\theta$ depends only on the difference $\alpha_{j}-\alpha_{l}$. This is accomplished by the map

$$
\begin{aligned}
z & =e^{i p}=\frac{e^{i \mu}-e^{\alpha}}{e^{i \mu+\alpha}-1} \\
\Delta & =-\cos \mu .
\end{aligned}
$$

This version of the map is appropriate for $-1<\Delta<1$. Some special values of $\alpha$ delineate the map: $\alpha=0$ corresponds to $e^{i p}=1$ which implies $p=0$, and $\alpha= \pm \infty$ map to $p= \pm(\pi-\mu)$. (We are choosing $p$ to be in the range $-\pi<p<\pi$.) Thus the whole range $-\infty<\alpha<\infty$ corresponds to $-(\pi-\mu)<p<\pi-\mu$. Note that $\Delta=1$ shrinks the range of $p$ to 0 , whereas $\Delta=-1$ represents the maximum range. It is straightforward to work out the following quantities in terms of the new variables:

$$
\begin{aligned}
\cos p & =-\cos \mu+\frac{\sin ^{2} \mu}{\cosh \alpha-\cos \mu} \\
\frac{d p}{d \alpha} & =\frac{\sin p}{\sinh \alpha}=\frac{\sin \mu}{\cosh \alpha-\cos \mu} \\
\theta(\alpha, \beta) & =2 \tan ^{-1}\left[(\cot \mu) \tanh \frac{\beta-\alpha}{2}\right]
\end{aligned}
$$

The boundary conditions take the form

$$
p_{l}=\frac{2 \pi I_{l}}{M}-\frac{1}{M} \sum_{j \neq l} \theta\left(\alpha_{j}, \alpha_{l}\right),
$$

where the $I_{l}$ are integers when $q$ is odd, and they are half-odd integers when $q$ is even. Different choices for these integers lead to different solutions for the set of $p$ 's.

\section{A.1 Consecutive $I_{l}: Q, P \neq 0$}

We begin by first choosing the set of numbers $I_{l}$ to be consecutive with no gaps: $I_{l+1}=1+I_{l}$. We define a kernel $K$ and density function $R(\alpha)$ by

$$
\begin{aligned}
K(\alpha, \beta) & \equiv \frac{1}{2 \pi} \frac{\partial \theta}{\partial \beta}=\frac{1}{2 \pi} \frac{\sin 2 \mu}{\cosh (\alpha-\beta)-\cos 2 \mu} \\
R(\alpha) & =\frac{2 \pi}{M} \frac{d j}{d \alpha}
\end{aligned}
$$

and then convert the equation for the $p$ 's as $M \rightarrow \infty$ into an integral equation

$$
\frac{d p}{d \alpha}=R(\alpha)+\int_{\alpha_{-}}^{\alpha_{+}} d \beta K(\alpha-\beta) R(\beta) .
$$


This equation was analyzed in $[18,19]$ for $\alpha_{-}=-\alpha_{+}$. The values chosen for $\alpha_{ \pm}$determine the characteristics of the eigenstate. For example, the eigenstate with the lowest energy corresponds to $\alpha_{ \pm}= \pm \infty$. The values of $p$ at the limits of this range are $p= \pm(\pi-\mu)$. As long as $0<\mu<\pi, e(\alpha)=4(\Delta-\cos p(\alpha))<0$ for all finite $\alpha$, so taking the whole range of $\alpha$ corresponds to including in the expression for $E$ all values for $e$ less than 0 . For the continuum limit we are only interested in very large $\alpha_{ \pm}$since then the eigenvalues will be close (within $1 / M$ ) of the minimum energy eigenvalue.

As shown in $[18,19]$, the kernel $J=-(I+K)^{-1} K$, can be used to rewrite the equation for $R$, which determines it over the whole range of $\alpha$, in terms of its values outside the range $\left(\alpha_{-}, \alpha_{+}\right)$. This is useful since we are interested only in the excited states close to the ground state corresponding to $\alpha_{ \pm}= \pm \infty$.

$$
R(\alpha)=R_{0}(\alpha)-\left[\int_{-\infty}^{\alpha_{-}}+\int_{\alpha_{+}}^{\infty}\right] J(\alpha-\beta) R(\beta)
$$

where $R_{0}$ is the solution of the equation for $\alpha_{ \pm}= \pm \infty$. It can be easily found by Fourier transformation of the equation. From

$$
\begin{aligned}
\frac{d p}{d \alpha} & =\int d \lambda e^{-i \lambda \alpha} \frac{\sinh (\pi-\mu) \lambda}{\sinh \pi \lambda} \\
K(\alpha) & =\int_{-\infty}^{\infty} \frac{d \lambda}{2 \pi} e^{-i \lambda \alpha} \frac{\sinh (\pi-2 \mu) \lambda}{\sinh \pi \lambda}
\end{aligned}
$$

we determine

$$
\begin{aligned}
R_{0}(\alpha) & =\int_{-\infty}^{\infty} d \lambda e^{-i \lambda \alpha} \frac{1}{2 \cosh \mu \lambda} \\
& =\frac{\pi}{2 \mu} \frac{1}{\cosh (\pi \alpha /(2 \mu))} .
\end{aligned}
$$

We can also easily express $J$ as a Fourier integral:

$$
J(\alpha)=-\int_{-\infty}^{\infty} \frac{d \lambda}{2 \pi} e^{-i \lambda \alpha} \frac{\sinh (\pi-2 \mu) \lambda}{2 \sinh (\pi-\mu) \lambda \cosh \mu \lambda} .
$$

The conserved quantities $Q=M-2 q, P=\sum_{j} p_{j}$, the total charge and total momentum respectively can be expressed, in the limit $M \rightarrow \infty$, as integrals either inside or outside the range $\left(\alpha_{-}, \alpha_{+}\right)$. These expressions then implicitly determine $\alpha_{ \pm}$in terms of $Q, P$.

$$
\begin{aligned}
\frac{1}{2}-\frac{Q}{2 M}=\frac{q}{M} & =\int_{\alpha_{-}}^{\alpha_{+}} \frac{d \beta}{2 \pi} R(\beta) \\
& =\int_{-\infty}^{\infty} \frac{d \beta}{2 \pi} R(\beta)-\left[\int_{-\infty}^{\alpha_{-}}+\int_{\alpha_{+}}^{\infty}\right] \frac{d \beta}{2 \pi} R(\beta) \\
& =\frac{1}{2}-\left[\int_{-\infty}^{\alpha_{-}}+\int_{\alpha_{+}}^{\infty}\right] \frac{d \beta}{2 \pi} R(\beta)\left(1+\int_{-\infty}^{\infty} d \alpha J(\alpha-\beta)\right)
\end{aligned}
$$

Now,

$$
1+\int_{-\infty}^{\infty} d \alpha J(\alpha-\beta)=1-\frac{\pi-2 \mu}{2(\pi-\mu)}=\frac{\pi}{2(\pi-\mu)}
$$


so we have

$$
\frac{Q}{M}=\frac{\pi}{\pi-\mu}\left[\int_{-\infty}^{\alpha_{-}}+\int_{\alpha_{+}}^{\infty}\right] \frac{d \beta}{2 \pi} R(\beta) .
$$

In a similar manner we can express the total momentum as

$$
\begin{aligned}
\frac{P}{M}=\frac{1}{M} \sum_{j=1}^{q} p_{j} & =\int_{\alpha_{-}}^{\alpha_{+}} \frac{d \beta}{2 \pi} R(\beta) p(\beta) \\
& =\int_{-\infty}^{\infty} \frac{d \beta}{2 \pi} R(\beta) p(\beta)-\left[\int_{-\infty}^{\alpha_{-}}+\int_{\alpha_{+}}^{\infty}\right] \frac{d \beta}{2 \pi} R(\beta) p(\beta) \\
& =\frac{P_{0}}{M}-\left[\int_{-\infty}^{\alpha_{-}}+\int_{\alpha_{+}}^{\infty}\right] \frac{d \beta}{2 \pi} R(\beta)\left(p(\beta)+\int_{-\infty}^{\infty} d \alpha J(\alpha-\beta) p(\alpha)\right)
\end{aligned}
$$

We can infer the Fourier transform of $p(\alpha)$ from that of $d p / d \alpha$.

$$
\begin{aligned}
\frac{d p}{d \alpha} & =\int d \lambda e^{-i \lambda \alpha} \frac{\sinh (\pi-\mu) \lambda}{\sinh \pi \lambda} \\
p(\beta) & =-\frac{1}{2 i} \int d \lambda e^{-i \lambda \beta} \frac{\sinh (\pi-\mu) \lambda}{\sinh \pi \lambda}\left[\frac{1}{\lambda+i \epsilon}+\frac{1}{\lambda-i \epsilon}\right] \\
p(\beta)+\int d \alpha J(\alpha-\beta) p(\alpha) & =-\frac{1}{2 i} \int d \lambda e^{-i \lambda \beta} \frac{1}{2 \cosh \mu \lambda}\left[\frac{1}{\lambda+i \epsilon}+\frac{1}{\lambda-i \epsilon}\right] \\
& \rightarrow \pm \frac{\pi}{2}, \quad \text { for } \quad \beta \rightarrow \pm \infty .
\end{aligned}
$$

Note that the $i \epsilon$ prescription is chosen so that $p( \pm \infty)= \pm(\pi-\mu)$, as required by the mapping. Finally, since $P_{0}=0$, we have for large $\alpha_{+}, \alpha_{-}$,

$$
\frac{P}{M} \approx-\frac{\pi}{2}\left[\int_{\alpha_{+}}^{\infty}-\int_{-\infty}^{\alpha_{-}}\right] \frac{d \beta}{2 \pi} R(\beta) .
$$

Finally, we manipulate the expression for the energy, expressing it as an integral outside the interval $\left(\alpha_{-}, \alpha_{+}\right)$:

$$
\begin{aligned}
\frac{E}{M}+\Delta & =\int_{\alpha_{-}}^{\alpha_{+}} \frac{d \beta}{2 \pi} R(\beta) e(\beta) \\
& \left.=\int_{-\infty}^{\infty} \frac{d \beta}{2 \pi} R(\beta) e(\beta)-\left[\int_{-\infty}^{\alpha_{-}}+\int_{\alpha_{+}}^{\infty}\right] \frac{d \beta}{2 \pi} R(\beta) e(\beta)\right] \\
& =\frac{E_{0}}{M}+\Delta-\left[\int_{-\infty}^{\alpha_{-}}+\int_{\alpha_{+}}^{\infty}\right] \frac{d \beta}{2 \pi} R(\beta)\left(e(\beta)+\int_{-\infty}^{\infty} d \alpha J(\alpha-\beta) e(\alpha)\right),
\end{aligned}
$$

where we have defined

$$
\begin{aligned}
e(\alpha) & =4 \Delta-4 \cos p=\frac{-4 \sin ^{2} \mu}{\cosh \alpha-\cos \mu}=-4 \sin \mu \frac{d p}{d \alpha} \\
& =-4 \sin \mu \int d \lambda e^{-i \lambda \alpha \frac{\sinh (\pi-\mu) \lambda}{\sinh \pi \lambda}}
\end{aligned}
$$


where the last line gives the Fourier transform of $e(\alpha)$. Also $E_{0}$ is the energy when $\alpha_{ \pm} \rightarrow \infty$ and $M$ is large:

$$
\begin{aligned}
\frac{E_{0}}{M}+\Delta & =\int_{-\infty}^{\infty} \frac{d \beta}{2 \pi} R_{0}(\beta) e(\beta) \\
& =-4 \sin ^{2} \mu \int_{-\infty}^{\infty} \frac{d \beta}{2 \pi} \frac{\pi}{2 \mu} \frac{1}{\cosh (\pi \beta /(2 \mu))(\cosh \beta-\cos \mu)}
\end{aligned}
$$

This integral is not elementary for general $\mu$. But for $\Delta=0(\mu=\pi / 2)$ it is easily done;

$$
\int_{-\infty}^{\infty} \frac{d \beta}{2 \pi} \frac{1}{\cosh ^{2} \beta}=\frac{1}{\pi}
$$

so that $E_{0} \rightarrow-4 M / \pi$ for $\mu \rightarrow \pi / 2$ or $\delta \rightarrow 0$. Comparison to our explicit evaluation at $\Delta=0$ shows that the correction $-4 \pi / 6 M$ is not included in $E_{0}$. That is because this term is the $M^{-1}$ correction to the replacement of the sum over discrete momenta by an integral.

We finally arrive at a convenient expression for $E-E_{0}$

$$
\begin{aligned}
\frac{E-E_{0}}{M} & =+4 \sin \mu\left[\int_{-\infty}^{\alpha_{-}}+\int_{\alpha_{+}}^{\infty}\right] \frac{d \beta}{2 \pi} R(\beta) \int_{-\infty}^{\infty} d \lambda e^{-i \lambda \beta} \frac{1}{2 \cosh \mu \lambda} \\
& =\frac{2 \pi \sin \mu}{\mu}\left[\int_{-\infty}^{\alpha_{-}}+\int_{\alpha_{+}}^{\infty}\right] \frac{d \beta}{2 \pi} R(\beta) \frac{1}{\cosh (\pi \beta / 2 \mu)},
\end{aligned}
$$

To find the energy levels close to the ground state, we must analyze the equations for $R$ for large $\alpha_{+}, \alpha_{-}$. For $\alpha>\alpha_{+}$, (A.6) can be approximated by dropping the integral over negative $\alpha$ and using the asymptotic form for $R_{0}$ :

$$
R(\alpha)+\int_{\alpha_{+}}^{\infty} J(\alpha-\beta) R(\beta) \approx \frac{\pi}{\mu} e^{-\pi \alpha / 2 \mu}
$$

It is convenient to put

$$
R\left(\alpha+\alpha^{+}\right)=\frac{\pi}{\mu} e^{-\pi \alpha_{+} / 2 \mu} S(\alpha)
$$

so that (A.23) reduces to the Wiener-Hopf equation [18, 19]

$$
S(\alpha)+\int_{0}^{\infty} J(\alpha-\beta) S(\beta)=e^{-\pi \alpha / 2 \mu} .
$$

Similarly, analyzing the equation for $\alpha<\alpha_{-}$, leads to the identification

$$
R\left(\alpha+\alpha^{-}\right) \approx \frac{\pi}{\mu} e^{\pi \alpha_{-} / 2 \mu} S(-\alpha) .
$$

Inserting these approximations into the formulas for $Q, P$, and $E$, leads to

$$
\begin{aligned}
\frac{Q}{M} & \approx \frac{\pi}{\pi-\mu} \frac{1}{2 \mu}\left[e^{-\pi \alpha_{+} / 2 \mu}+e^{\pi \alpha_{-} / 2 \mu}\right] \int_{0}^{\infty} d \beta S(\beta) \\
\frac{P}{M} & \approx-\frac{\pi}{2} \frac{1}{2 \mu}\left[e^{-\pi \alpha_{+} / 2 \mu}-e^{\pi \alpha_{-} / 2 \mu}\right] \int_{0}^{\infty} d \beta S(\beta) \\
\frac{E-E_{0}}{M} & \approx \frac{2 \pi \sin \mu}{\mu} \frac{1}{\mu}\left[e^{-\pi \alpha_{+} / \mu}+e^{\pi \alpha_{-} / \mu}\right] \int_{0}^{\infty} d \beta S(\beta) e^{-\pi \beta / 2 \mu} .
\end{aligned}
$$


Next one can solve the first two equations for $\alpha_{+}$and $\alpha_{-}$and substitute in the last equation to get

$$
\frac{E-E_{0}}{M} \approx \frac{2 \pi \sin \mu}{\mu} \frac{2 \mu I(\pi / 2 \mu)}{I(0)^{2}}\left[\frac{(\pi-\mu)^{2}}{\pi^{2}} \frac{Q^{2}}{M^{2}}+\frac{4}{\pi^{2}} \frac{P^{2}}{M^{2}}\right]
$$

where we have defined $I(x)=\int_{0}^{\infty} d \beta S(\beta) e^{-x \beta}$. From the solution of (A.24), one can infer (see $[18,19])$ that $I(\pi / 2 \mu) / I(0)^{2}=\pi^{2} / 8 \mu(\pi-\mu)$, so finally

$$
E-E_{0} \approx \frac{2 \pi \sin \mu}{\mu} \frac{1}{M}\left[\frac{\pi-\mu}{4} Q^{2}+\frac{1}{\pi-\mu} P^{2}\right]
$$

\section{A.2 Non-consecutive $I_{l}$}

The excited states included in (A.27) are those where the numbers $I_{l}$ are consecutive. For example, the state with $Q=P=0$ corresponds to the choice (with $q=M / 2$ odd)

$$
\left(-\frac{q-1}{2}, \ldots, \frac{q-3}{2}, \frac{q-1}{2}\right) \text {. }
$$

There are also excitations in which "holes" are allowed in this set of numbers. As an example, consider replacing $(q-1-2 j) / 2$ in the above list by $(q+1) / 2$, creating a gap, but retaining the same number of overturned arrows, so that $Q=0$. However the momentum is increased by the amount $P=2 \pi(j+1) / M$. For large $M$, the effect of this hole on the $p$ 's is small, and it makes sense to expand them around the values appropriate to the $Q=P=0$ state, the new set of $p$ 's differing from the latter by $\delta p_{j}$. Referring to the original equation for the $p$ 's, we find an equation for $\delta p$ :

$$
\begin{aligned}
\delta p_{l} & =\frac{2 \pi}{M} \theta\left(l-l_{j}\right)+\frac{2 \pi}{M} \sum_{j \neq l}\left[-\frac{\partial \alpha_{j}}{\partial p_{j}} \delta p_{j}+\frac{\partial \alpha_{l}}{\partial p_{l}} \delta p_{l}\right] K\left(\alpha_{l}-\alpha_{j}\right) \\
\delta p_{l}\left(1-\frac{\partial \alpha_{l}}{\partial p_{l}} \frac{2 \pi}{M} \sum_{j \neq l} K\left(\alpha_{l}-\alpha_{j}\right)\right) & =\frac{2 \pi}{M} \theta\left(l-l_{j}\right)-\frac{2 \pi}{M} \sum_{j \neq l}\left[\frac{\partial \alpha_{j}}{\partial p_{j}} \delta p_{l}\right] K\left(\alpha_{l}-\alpha_{j}\right) \\
\delta p(\alpha) \frac{\partial \alpha}{\partial p} R(\alpha) & =\frac{2 \pi}{M} \theta\left(\alpha-\alpha_{j}\right)-\int d \beta \frac{\partial \beta}{\partial p} \delta p(\beta) R(\beta) K(\alpha-\beta),(\mathrm{A} .28
\end{aligned}
$$

where we have replaced the sums by integrals in the last line. Defining

$$
\chi(\alpha)=M \delta p(\alpha) R(\alpha) d \alpha / d p,
$$

we have the integral equation

$$
\chi(\alpha)+\int_{-\infty}^{\infty} d \beta K(\alpha-\beta) \chi(\beta)=2 \pi \theta\left(\alpha-\alpha_{j}\right) .
$$

Here $\alpha_{j}$ marks the location of the "hole". It can be related to the value for the momentum of the excited state:

$$
P=\frac{2 \pi(j+1)}{M}=\sum_{l} \delta p_{l}=\int_{-\infty}^{\infty} \frac{d \alpha}{2 \pi} \chi(\alpha) \frac{d p}{d \alpha}
$$


Similarly, we can write the energy difference between the excited and ground state as

$$
\begin{aligned}
E-E_{0} & =\sum_{l} \delta p_{l} \frac{d \alpha_{l}}{d p_{l}} \frac{d e\left(\alpha_{l}\right)}{d \alpha_{l}} \\
& =-4 \sin \mu \int_{-\infty}^{\infty} \frac{d \alpha}{2 \pi} \chi(\alpha)\left[\int d \lambda e^{-i \lambda \alpha} \frac{-i \lambda \sinh (\pi-\mu) \lambda}{\sinh \pi \lambda}\right]
\end{aligned}
$$

(A.29) can be immediately solved via Fourier transformation:

$$
\chi(\alpha)=i \int d \lambda e^{-i\left(\alpha-\alpha_{j}\right) \lambda} \frac{\sinh \pi \lambda}{2(\lambda+i \epsilon) \sinh (\pi-\mu) \lambda \cosh \mu \lambda},
$$

and used to obtain the total momentum and energy

$$
\begin{aligned}
P & =\frac{i}{2} \int d \lambda e^{-i \lambda \alpha_{j}} \frac{1}{(-\lambda+i \epsilon) \cosh \mu \lambda} \\
E-E_{0} & =2 \sin \mu \int d \lambda e^{-i \lambda \alpha_{j}} \frac{1}{\cosh \mu \lambda} .
\end{aligned}
$$

Of course, we are interested in these expressions in the limit $\alpha_{j} \rightarrow \infty$, corresponding to the continuum limit. This asymptotic limit is obtained by deforming the integration contours into the lower half plane and picking up the nearest pole to the real axis, namely the one at $\lambda=-i \pi / 2 \mu$. This leads to

$$
P \sim 2 e^{-\pi \alpha_{j} / 2 \mu} \quad E-E_{0} \sim 2 \frac{2 \pi \sin \mu}{\mu} e^{-\pi \alpha_{j} / 2 \mu}
$$

from which we conclude that

$$
E-E_{0}=\frac{2 \pi \sin \mu}{\mu} P=\frac{2 \pi \sin \mu}{\mu} \frac{2 \pi(j+1)}{M}
$$

in the limit $M \rightarrow \infty$. Notice the important fact that the energy of these excitations is the factor $(2 \pi \sin \mu) / \mu$, common to the other contributions to $E$, times a coefficient independent of $\mu$. Although we have discussed only one particular "particle-hole" excitation, it is clear that the energy of the state with many particle-hole pairs will simply be additive in the momentum carried by each pair. Furthermore, there are two independent sets of such excitations about the two boundaries of the Fermi sea. Each particle hole excitation contributes $2 \pi n T_{0} / P^{+}$, where $n>0$. If there are several particle-hole pairs from the right side $p>0$ of the Fermi sea, we define $N_{R}=\sum_{i} n_{i}$, and similarly $N_{L}$ is defined for those from the left side $p<0$ of the Fermi sea.

These contributions to the energy are added to those arising from non-zero $Q, P$. Note that the $P^{2}$ term in the energy receives negligible contributions from particle-hole excitations from the same side of the Fermi sea, since these have $P=O(1 / M)$. This term is non-zero in the continuum limit only if the particle and hole are from opposite sides of the sea. For example, replacing $-(q+1) / 2$ with $(q+1) / 2$ contributes $2 \pi q / M \approx \pi$ to $P$. But such large momentum pair excitations have already been accounted for among the 
excitations with consecutive $I_{l}$ considered earlier. Thus the energy levels of the continuum limit are determined by $Q, P, N_{R}$, and $N_{L}$ :

$$
E-E_{0}=\frac{2 \pi \sin \mu}{\mu}\left[-\frac{\pi}{6}+\frac{\pi-\mu}{4} Q^{2}+\frac{1}{\pi-\mu} P^{2}+2 \pi\left(N_{R}+N_{L}\right)\right] \frac{1}{M} .
$$

Recall that $Q=2 r$ and $P=\pi s$ where $r, s$ range independently over all integers. Here we have taken the liberty of inserting the $-\pi /(6 M)$ correction, whose value should be the same relative to $2 \pi\left(N_{R}+N_{L}\right)$ as in the $\Delta=0$ case. This is because this term is just the zero point energy associated with the particle hole excitations [29].

Open Access. This article is distributed under the terms of the Creative Commons Attribution License (CC-BY 4.0), which permits any use, distribution and reproduction in any medium, provided the original author(s) and source are credited.

\section{References}

[1] C.B. Thorn, Reformulating string theory with the $1 / N$ expansion, in Sakharov memorial lectures in physics, vol. 1, L.V. Keldysh and V.Ya. Fainberg eds., Nova Science Publishers, Commack U.S.A. (1992), pg. 447 [hep-th/9405069] [INSPIRE].

[2] O. Bergman and C.B. Thorn, String bit models for superstring, Phys. Rev. D 52 (1995) 5980 [hep-th/9506125] [INSPIRE].

[3] C.B. Thorn, Substructure of string, hep-th/9607204 [INSPIRE].

[4] G. 't Hooft, A planar diagram theory for strong interactions, Nucl. Phys. B 72 (1974) 461 [INSPIRE].

[5] C.B. Thorn, A Fock space description of the $1 / N_{c}$ expansion of quantum chromodynamics, Phys. Rev. D 20 (1979) 1435 [inSPIRE].

[6] G. 't Hooft, Quantization of discrete deterministic theories by Hilbert space extension, Nucl. Phys. B 342 (1990) 471 [inSPIRE].

[7] G. 't Hooft, On the quantization of space and time, in Proc. of the $4^{\text {th }}$ Seminar on Quantum Gravity, Moscow USSR May 25-29 1987, M.A. Markov ed., World Scientific Press, Singapore (1988) [INSPIRE].

[8] G. 't Hooft, Dimensional reduction in quantum gravity, gr-qc/9310026 [INSPIRE].

[9] S. Sun and C.B. Thorn, Stable string bit models, Phys. Rev. D 89 (2014) 105002 [arXiv: 1402.7362] [INSPIRE].

[10] S. Sun, Detailed study of the simplest superstring bit model, to appear.

[11] G. 't Hooft, On the foundations of superstring theory, Found. Phys. 43 (2013) 46 [InSPIRE].

[12] P. Goddard, C. Rebbi and C.B. Thorn, Lorentz covariance and the physical states in dual resonance models, Nuovo Cim. A 12 (1972) 425 [INSPIRE].

[13] P. Goddard, J. Goldstone, C. Rebbi and C.B. Thorn, Quantum dynamics of a massless relativistic string, Nucl. Phys. B 56 (1973) 109 [INSPIRE].

[14] S. Mandelstam, Interacting string picture of dual resonance models, Nucl. Phys. B 64 (1973) 205 [inSPIRE]. 
[15] S. Mandelstam, Interacting string picture of the Neveu-Schwarz-Ramond model, Nucl. Phys. B 69 (1974) 77 [INSPIRE].

[16] R. Giles and C.B. Thorn, A lattice approach to string theory, Phys. Rev. D 16 (1977) 366 [INSPIRE].

[17] H. Bethe, On the theory of metals. 1. Eigenvalues and eigenfunctions for the linear atomic chain (in German), Z. Phys. 71 (1931) 205 [InSPIRE].

[18] C.-N. Yang and C.P. Yang, One-dimensional chain of anisotropic spin spin interactions. 1. Proof of Bethe's hypothesis for ground state in a finite system, Phys. Rev. 150 (1966) 321 [INSPIRE].

[19] C.N. Yang and C.P. Yang, One-dimensional chain of anisotropic spin spin interactions. 2. Properties of the ground state energy per lattice site for an infinite system, Phys. Rev. 150 (1966) 327 [InSPIRE].

[20] F. Gliozzi, J. Scherk and D.I. Olive, Supersymmetry, supergravity theories and the dual spinor model, Nucl. Phys. B 122 (1977) 253 [INSPIRE].

[21] P. Ramond, Dual theory for free fermions, Phys. Rev. D 3 (1971) 2415 [inSPIRE].

[22] A. Neveu and J.H. Schwarz, Factorizable dual model of pions, Nucl. Phys. B 31 (1971) 86 [INSPIRE].

[23] A. Neveu, J.H. Schwarz and C.B. Thorn, Reformulation of the dual pion model, Phys. Lett. B 35 (1971) 529 [inSPIRE].

[24] C.B. Thorn, Embryonic dual model for pions and fermions, Phys. Rev. D 4 (1971) 1112 [INSPIRE].

[25] A. Neveu and J.H. Schwarz, Quark model of dual pions, Phys. Rev. D 4 (1971) 1109 [INSPIRE].

[26] K. Bardakci and M.B. Halpern, New dual quark models, Phys. Rev. D 3 (1971) 2493 [INSPIRE].

[27] M.B. Green and J.H. Schwarz, Supersymmetrical dual string theory, Nucl. Phys. B 181 (1981) 502 [inSPIRE].

[28] R. Giles, L.D. McLerran and C.B. Thorn, The string representation for a field theory with internal symmetry, Phys. Rev. D 17 (1978) 2058 [InSPIRE].

[29] L. Brink and H.B. Nielsen, A simple physical interpretation of the critical dimension of space-time in dual models, Phys. Lett. B 45 (1973) 332 [INSPIRE].

[30] M.B. Green, J.H. Schwarz and L. Brink, Superfield theory of type II superstrings, Nucl. Phys. B 219 (1983) 437 [InSPIRE]. 